\title{
Deficiencia de hierro y anemia ferropénica. Guía para su prevención, diagnóstico y tratamiento
} Iron Deficiency and Iron Deficiency Anemia. Guideline for
Prevention, Diagnosis and Treatment

\author{
Comité Nacional de Hematología, Oncología y Medicina Transfusional y \\ Comité Nacional de Nutrición
}

a. Hospital del Niño de San Justo, Sección Hematología/

Oncología, San Justo, Buenos Aires.

b. Consultorios de Hematología Infantil, Ciudad Autónoma de Buenos Aires.

c. Comité de Nutrición, Sociedad Argentina de Pediatría, Buenos Aires.

d. Hospital Pediátrico A. Fleming, Servicio de Hematología, ciudad de Mendoza.

e. Hospital L. Lagomaggiore, Hematología Neonatal, ciudad de Mendoza.

f. Sanatorio San Lucas, Hematología Infantil, ciudad de Neuquén.

g. Hospital de Niños V. J. Vilela, Servicio de Oncología, Rosario, Santa Fe.

h. Hospital Español, Servicio de Hematología y Oncología Pediátrica, Rosario, Santa Fe.

i. Hospital Italiano, Servicio de Hematología y Oncología Pediátrica, Rosario, Santa Fe.

j. Cátedra de Nutrición, Facultad de Farmacia y Bioquímica, Universidad de Buenos Aires.

Correspondencia: Dr. Hugo Donato: hcdonato@gmail.com

Financiamiento:

Ninguno.

Conflicto de intereses: Ninguno que declarar.

Recibido: 8-9-2016 Aceptado: 30-9-2016 http: / / dx.doi.org/10.5546/ aap.2017.s68

Resumen ejecutivo publicado en Arch Argent Pediatr 2017;115(4):406-408.

\section{RESUMEN}

La deficiencia de hierro es la causa más frecuente de anemia en el niño, especialmente, en edad preescolar, con una prevalencia mayor del $35 \%$ en menores de 24 meses, en Argentina. Su detección precoz, así como el tratamiento correcto y la profilaxis adecuada, son hoy una prioridad en nuestro país. Con dicho objetivo, en esta guía, se establece la definición de anemia según edad cronológica, edad gestacional y medioambiente; se enumeran las principales causas de deficiencia de hierro y se establecen pautas para su diagnóstico correcto, diagnóstico diferencial, tratamiento, prevención y pesquisa. Palabras clave: hierro, anemia, deficiencia de hierro, anemia ferropénica, vitamina $C$.

\section{ABSTRACT}

Iron deficiency is the most important causal factor of anemia. Preschoolers are particularly vulnerable; a recentanalysis reported a prevalence rate higher than $35 \%$ among children less than 2 years old in Argentina. Its early detection, right treatment, and suitable prophylaxis currently take priority in our country. This guideline establishes the definition of anemia in relation to chronological age, gestational age and habitat, reviews main causes of iron deficiency, and sets guidelines for diagnosis, detection, differential diagnosis, treatment, prevention and screening of iron deficiency.

Key words: iron, anemia, iron deficiency, iron deficiency anemia, vitamin $C$.

Cómo citar: Comité Nacional de Hematología, Oncología y Medicina Transfusional, Comité Nacional de Nutrición. Deficiencia de hierro y anemia ferropénica. Guía para su prevención, diagnóstico y tratamiento.. Arch Argent Pediatr 2017;115 Supl 4: s68-s82

\section{INTRODUCCIÓN}

La deficiencia de hierro y la anemia son problemas de salud pública universal por sus consecuencias sobre la salud de los individuos y sobre aspectos sociales y/o económicos, que afectan en distinto grado a todos los países. Ocurre a todas las edades, pero su prevalencia es máxima en niños pequeños y mujeres en edad fértil. Según datos de la Organización Mundial de la Salud (OMS) (2011), más de 2 billones de personas tienen deficiencia de hierro, lo que representa casi el 25\% de la población mundial. La anemia está presente en 800 millones de personas, y son niños 273 millones. Se estima que la sufren, aproximadamente, el $50 \%$ de los niños menores de 5 años y el $25 \%$ de los de 6-12 años de la población mundial (Tabla 1). ${ }^{1}$ La causa más frecuente de anemia en el mundo es la deficiencia de hierro; su incidencia en países en vías de desarrollo es 2,5 veces mayor que en países desarrollados. ${ }^{2} \mathrm{Se}$ presenta, especialmente, entre los 6 y los 24 meses de edad. De acuerdo con la Encuesta Nacional de Nutrición y Salud (2005), en Argentina, presentan anemia $16 \%$ de los menores de 5 años, $35 \%$ de los niños de 6-24 meses de
Coordinadores: Dr. Hugo Donato ${ }^{a, b}$ y Dra. Norma Piazza ${ }^{c}$

Colaboradores:Dra.María C. Rapetti ${ }^{a}$,Dra. Susana de Grandis ${ }^{c}$, Dra. Viviana Bacciedoni ${ }^{d,}$,

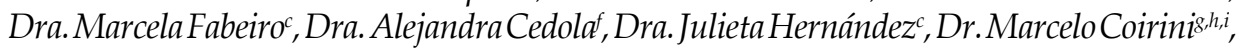
Dra. María L. Pita de Portela', Dra. Patricia Sosac y Dra. Virginia Desantadina ${ }^{c}$ 
edad y $20 \%$ de mujeres en edad fértil (Figura 1). ${ }^{3}$ Esta prevalencia varía en las distintas regiones y alcanza valores considerablemente mayores en las de peores condiciones socioeconómicas; p. ej., en el Noreste, la prevalencia de anemia en menores de 2 años llega a casi $46 \%$. Se han comunicado cifras aun más elevadas. Estudios de la década del noventa en niños de 6-24 meses mostraban prevalencias de $60 \%$ de deficiencia de hierro y $47 \%$ de anemia en el Gran Buenos Aires y de 66\% de anemia en Chaco. ${ }^{4,5}$

En nuestro país, se han tomado medidas de salud pública que han demostrado ser eficaces para disminuir la prevalencia de la deficiencia de hierro y la anemia, como la recomendación de ligadura tardía del cordón umbilical, las
Leyes 25459/01 y 25630/02 de fortificación de los alimentos con hierro, zinc y vitaminas, y el acceso gratuito al suplemento con sulfato ferroso de las mujeres embarazadas y los niños menores de 5 años que se atienden en los centros de atención primaria de la salud pública de todo el país a través del Programa REMEDIAR. Pero, si bien no se dispone de publicaciones que evalúen técnicamente la aplicación de los programas y su implementación en cada contexto de salud pública, algunas investigaciones parciales y locales demuestran la insuficiente indicación del sulfato ferroso como preventivo en la atención primaria, según la evaluación del stock y de las recetas usadas en el Programa REMEDIAR. ${ }^{6-8}$ En el mismo sentido, otros estudios señalan que el

Tabla 1. Prevalencia de anemia en la población mundial por grupo poblacional

\begin{tabular}{lcc}
\hline Grupo poblacional & Prevalencia $(\%)$ (media \pm DE) & Población afectada (millones) (media \pm DE) \\
\hline Niños - edad preescolar & $47,4 \pm 0,85$ & $293 \pm 5,0$ \\
Niños - edad escolar & $25,4 \pm 2,75$ & $305 \pm 33,2$ \\
Mujeres embarazadas & $41,8 \pm 0,95$ & $56 \pm 1,2$ \\
Mujeres en edad fértil & $30,2 \pm 0,72$ & $468 \pm 11,2$ \\
Hombres & $12,7 \pm 2,15$ & $260 \pm 42,5$ \\
Ancianos (> 60 años) & $23,9 \pm 2,75$ & $164 \pm 19,0$ \\
Total & $24,8 \pm 0,95$ & $1620 \pm 60$ \\
\hline
\end{tabular}

DE: desvío estándar.

Figura 1. Prevalencia de anemia en Argentina a distintas edades

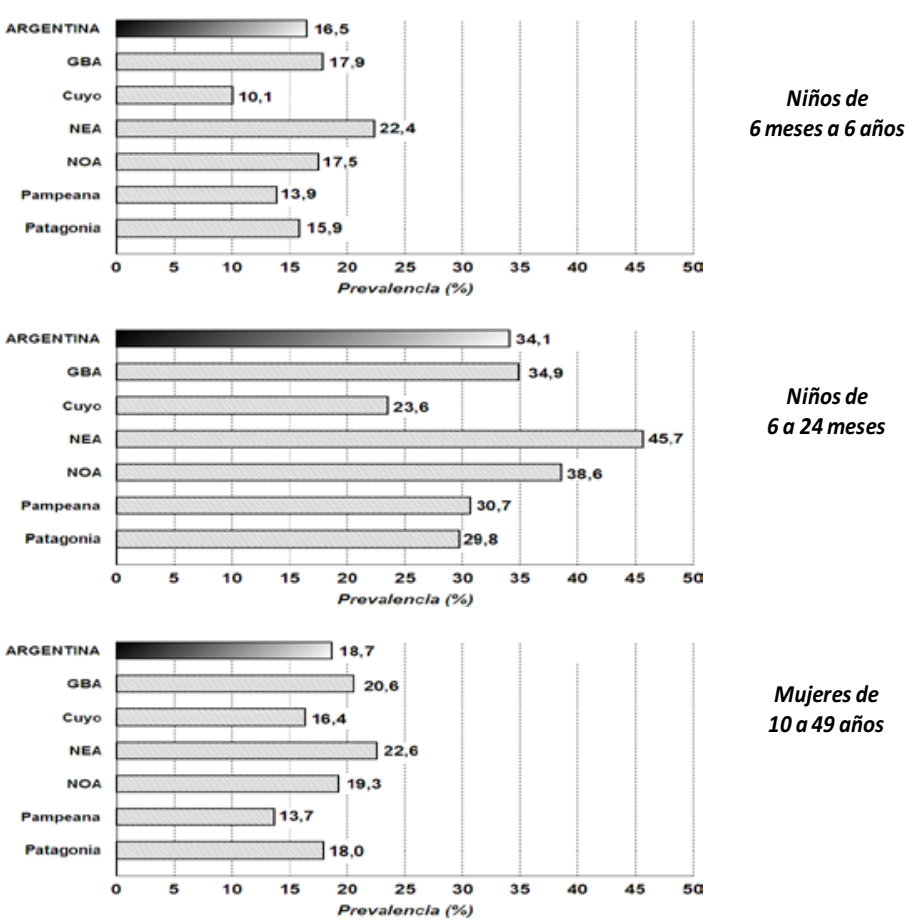

GBA: Gran Buenos Aires; NEA: Noreste Argentino; NOA: Noroeste Argentino. 
$32 \%$ de los niños de 6-24 meses no lo recibieron por falta de indicación médica. ${ }^{9}$

El recién nacido normal de término tiene reservas adecuadas de hierro, suficientes para cubrir los requerimientos hasta los 4-6 meses de edad. ${ }^{10}$ Estas provienen, fundamentalmente, del aporte de hierro materno durante la vida intrauterina $y$, en menor medida, del originado por la destrucción de los eritrocitos por envejecimiento durante los primeros 3 meses de vida. ${ }^{11}$ Como el hierro materno es incorporado por el feto durante el tercer trimestre del embarazo, el niño pretérmino nace con reservas disminuidas de hierro. Si bien, en la actualidad, está en discusión la influencia de la deficiencia materna sobre el estado del hierro en el neonato, la mayor evidencia parece mostrar que los hijos de madres con anemia ferropénica nacen con depósitos disminuidos de hierro. ${ }^{12,13} \mathrm{~A}$ partir de los 4-6 meses de vida, el niño depende, en gran medida, de la ingesta dietética para mantener un balance adecuado de hierro, ${ }^{11,14}$ por lo cual la deficiencia de hierro y la anemia ferropénica en el lactante y en la primera infancia, generalmente, están determinadas por una dieta insuficiente o mal balanceada. La introducción tardía de alimentos ricos en hierro de alta biodisponibilidad (carnes) y / o la incorporación temprana de leche de vaca -antes de los 6 meses de edad-y/o las papillas a base de tubérculos o cereales no fortificados son causas frecuentes e importantes. ${ }^{15,16}$ También es usual encontrar, en niños mayores, exceso de ingesta de leche y / o carbohidratos. Este tipo de alimentación, aunque pobre en hierro y otros nutrientes que intervienen en su absorción y metabolismo, es, por lo general, adecuada en calorías, lo que da como resultado un niño con deficiencia de hierro $\mathrm{o}$ anemia ferropénica, pero con peso normal, $\mathrm{u}$, ocasionalmente, sobrepeso, para su edad.

Las recomendaciones de esta guía están, en general, basadas en aspectos fisiopatológicos y en opiniones de expertos. No hay otros niveles de evidencia en la bibliografía mundial.

TABLA 2. Valores promedio normales de hemoglobina $(g / d L)$ durante los primeros 3 meses de vida de acuerdo con el peso de nacimiento

\begin{tabular}{lcccc}
\hline Edad & \multicolumn{4}{c}{ Peso de nacimiento } \\
& $\mathbf{< 1 0 0 0} \mathbf{g}$ & $\mathbf{1 0 0 1 - 1 5 0 0 ~} \mathbf{g}$ & $\mathbf{1 5 0 1 - 2 0 0 0} \mathbf{~}$ & $>\mathbf{2 0 0 0} \mathbf{~}$ \\
\hline Nacimiento & $16,5(13,5)$ & $16,5(13,5)$ & $16,5(13,5)$ & $16,5(13,5)$ \\
24 horas & $19,3(15,4)$ & $18,8(14,6)$ & $19,4(15,6)$ & $19,3(14,9)$ \\
2 semanas & $16,0(13,6)$ & $16,3(11,3)$ & $14,8(11,8)$ & $16,6(13,4)$ \\
1 mes & $10,0(6,8)$ & $10,9(8,7)$ & $11,5(8,2)$ & $13,9(10,0)$ \\
2 meses & $8,0(7,1)$ & $8,8(7,1)$ & $9,4(8,0)$ & $11,2(9,4)$ \\
3 meses & $8,9(7,9)$ & $9,8(8,9)$ & $10,2(9,3)$ & $11,5(9,5)$ \\
\hline
\end{tabular}

Los valores entre paréntesis expresan el límite inferior normal (media - 2 DE).

DE: desvío estándar.

TABLA 3. Valores normales de hemoglobina y hematocrito durante la infancia y la adolescencia

\begin{tabular}{lcc}
\hline Edad & Hemoglobina (g/dL) & Hematocrito (\%) \\
\hline De 6 a 23 meses & $12,5(11,0)$ & $37(33)$ \\
De 2 a 4 años & $12,5(11,0)$ & $38(34)$ \\
De 5 a 7 años & $13,0(11,5)$ & $39(35)$ \\
De 8 a 11 años & $13,5(12,0)$ & $40(36)$ \\
De 12 a 14 años & & \\
$\quad$ Mujer & $13,5(12,0)$ & $41(36)$ \\
$\quad$ Varón & $14,0(12,5)$ & $43(37)$ \\
De 15 a 17 años & & \\
$\quad$ Mujer & $14,0(12,0)$ & $41(36)$ \\
$\quad$ Varón & $15,0(13,0)$ & $46(38)$ \\
Adulto & & $42(37)$ \\
$\quad$ Mujer & $14,0(12,0)$ & $47(40)$ \\
$\quad$ Varón & $16,0(14,0)$ &
\end{tabular}

Los valores entre paréntesis expresan el límite inferior normal (media - $2 \mathrm{DE}$ ).

DE: desvío estándar. 


\section{DEFINICIÓN}

Se define anemia como `disminución de la masa de glóbulos rojos y / o de la concentración de hemoglobina por debajo del segundo desvío estándar respecto de la media para edad y sexó (se debe tener en cuenta que, sobre la base de esta definición, se diagnosticarán como anémicos un 2,5\% de niños normales). ${ }^{17}$ En las Tablas 2 y 3 , se muestran los valores normales a distintas edades. ${ }^{11,17-20}$ Para poblaciones que viven en la altura, se debe calcular que la concentración normal de hemoglobina aumenta en 1,52 g/ dL por cada $1000 \mathrm{~m}$ que se ascienden sobre el nivel del mar (s. n. m.) (Sociedad Panamericana de la Salud, Reunión de Expertos, Lima, Perú, noviembre de 2011).

\section{CAUSAS}

El estado nutricional de hierro de una persona depende del balance determinado por la interacción entre los nutrientes que componen la dieta, la biodisponibilidad, las pérdidas y los requerimientos por crecimiento. ${ }^{10,21,22} \mathrm{La}$ cantidad de hierro que asimila el organismo depende de la cantidad ingerida, la composición de la dieta y la regulación de la absorción por la mucosa intestinal. ${ }^{21,23,24} \mathrm{La}$ biodisponibilidad

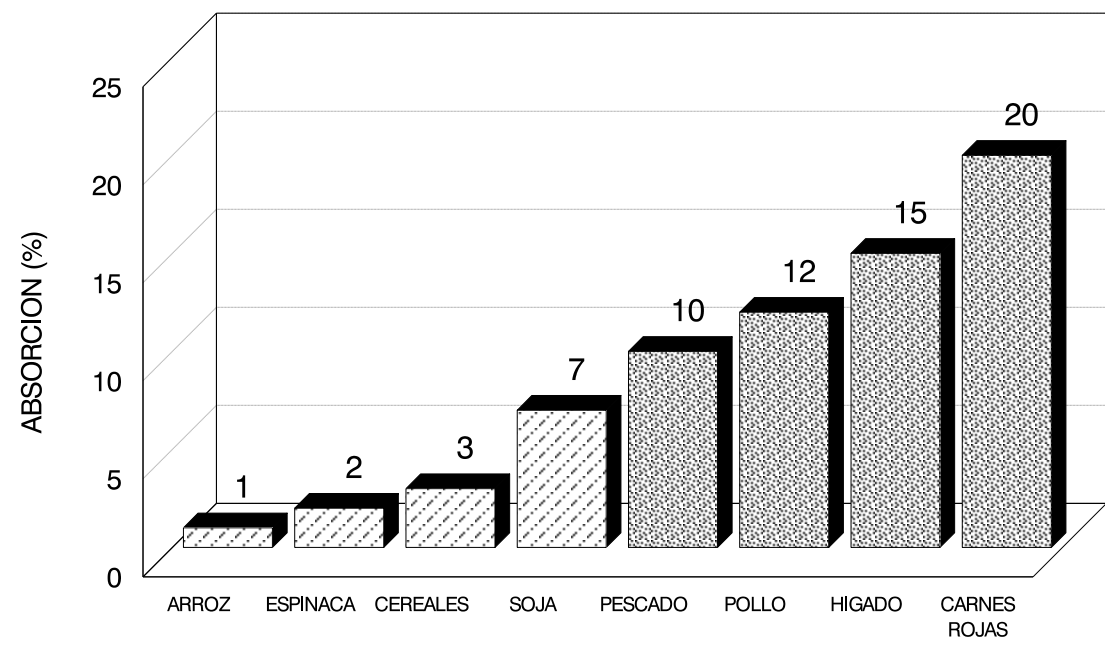

FIGURA 3. Biodisponibilidad del hierro en distintas leches

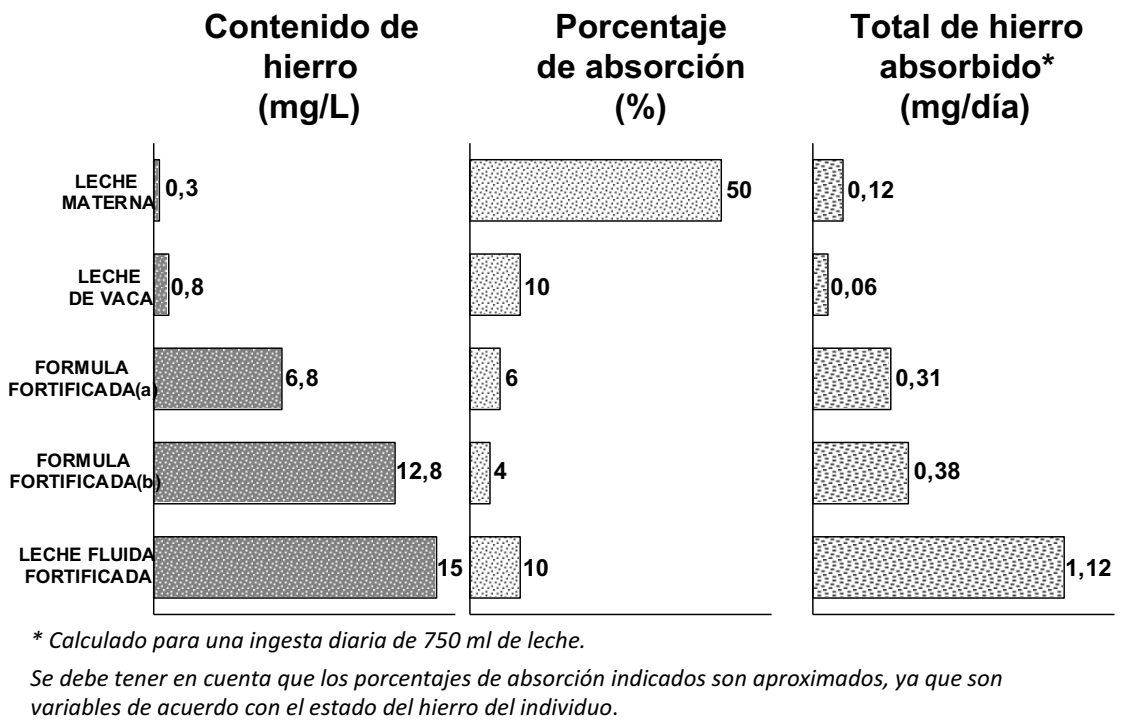


depende del estado químico en que se encuentra (hemo o no-hemo) y de su interrelación con otros componentes de la dieta, facilitadores (ácido ascórbico, fructosa, ácido cítrico, ácido láctico, factor cárneo) o inhibidores (fosfatos, fitatos, calcio, fibras, oxalatos, tanatos, polifenoles) de la absorción. ${ }^{23,25,26} \mathrm{El}$ hierro hemo es el de mejor disponibilidad, pues es absorbido sin sufrir modificaciones y sin interrelacionar con otros componentes de la dieta. Por tanto, los alimentos que más hierro aportan son los de origen animal (Figura 2). ${ }^{22}$ En las leches, su contenido y biodisponibilidad varía enormemente; la leche materna, con el menor contenido de hierro, presenta la máxima absorción alrededor del 50\%-.27-30 En la Figura 3, se muestra la biodisponibilidad del hierro presente en distintos tipos de leche. ${ }^{11,22,27,28,31-34}$ Los niños alimentados a pecho o con fórmulas tienen cubierto su requerimiento diario mínimo, no así los alimentados con leche de vaca no fortificada con hierro. ${ }^{11,15,35} \mathrm{La}$ absorción de hierro por la mucosa intestinal está regulada por la cantidad de hierro corporal y el ritmo de eritropoyesis. ${ }^{36} \mathrm{La}$ eritropoyesis depende también de muchos otros factores; p. ej., la vitamina A para la movilización de hierro desde los depósitos o la relación hierro/ proteínas para el transporte.
En la Tabla 4, se muestran valores promedio de requerimientos e ingesta a distintas edades. . $^{11,37,38}$ Como se observa, hay períodos de la vida en que este balance es negativo, por lo que el organismo debe recurrir al hierro de depósito para sostener una eritropoyesis adecuada. Durante estos, una dieta con insuficiente cantidad o baja biodisponibilidad de hierro agrava el riesgo de desarrollar deficiencia de hierro o anemia ferropénica. Estos períodos críticos son, fundamentalmente, tres: ${ }^{22}$

a. Primer año de vida: Los requerimientos por crecimiento son máximos, mientras que la ingesta es relativamente pobre.

\section{b. Adolescencia:}

- Varones: Los requerimientos por crecimiento son elevados y la dieta puede no aportar hierro suficiente.

- Mujeres: A los elevados requerimientos por crecimiento, se agregan las pérdidas menstruales. Como agravante, la dieta, por motivos socioculturales, suele ser marcadamente deficiente en hierro.

c. Embarazo: Los requerimientos son elevados, desde $1 \mathrm{mg} / \mathrm{kg} /$ día al comienzo a $6 \mathrm{mg} / \mathrm{kg} /$ día en el tercer trimestre.

En la Tabla 5, se muestran los requerimientos diarios mínimos recomendados en la actualidad. ${ }^{39}$

TABLA 4. Variación de la ingesta y los requerimientos de hierro en distintas etapas de la vida

\begin{tabular}{lccccc}
\hline Edad (años) & \multicolumn{3}{c}{ Requerimientos de hierro (mg/día) } & \multicolumn{2}{c}{$\begin{array}{c}\text { Ingesta de hierro } \\
\text { (mg/día) }\end{array}$} \\
\cline { 2 - 5 } & Pérdida & Crecimiento & Menstruación & Total & 6 \\
\hline 1 & 0,25 & 0,80 & - & 1,05 & 9 \\
13 (varón) & 0,33 & 0,30 & - & 0,63 & 17 \\
13 (mujer) & 0,80 & 0,50 & - & 1,30 & 15 \\
Adulto (varón) & 0,80 & 0,50 & 0,60 & 1,90 & 18 \\
Adulto (mujer) & 1,00 & - & - & 1,00 & 16 \\
Embarazada & 1,00 & - & 0,60 & 1,60 & 15 \\
\hline
\end{tabular}

* Se absorbe, aproximadamente, el 10\%.

TABLA 5. Ingestas dietéticas de referencia

\begin{tabular}{lcc}
\hline Grupo etario & Ingesta recomendada (mg/día) & Ingesta máxima tolerable (mg/día) \\
\hline Lactantes & 0,27 & 40 \\
0-6 meses & 11 & 40 \\
7-12 meses & & 40 \\
Niños & 7 & 40 \\
$1-3$ años & 10 & 40 \\
$4-8$ años & 8 & 45 \\
9-13 años & 11 & 45 \\
Adolescentes & 15 & 45 \\
$\quad$ Varones & 27 & 45 \\
Mujeres & 10 & \\
Embarazadas & & \\
Lactancia &
\end{tabular}


En la Tabla 6, se enumeran las causas de deficiencia de hierro. ${ }^{22}$

\section{MANIFESTACIONES CLÍNICAS}

La deficiencia de hierro es una enfermedad sistémica que afecta a múltiples órganos y tejidos, lo que hace que sus manifestaciones clínicas puedan ser muy variadas. La anemia es la forma más frecuente de presentación de la enfermedad y la de mayor facilidad para el diagnóstico, ya que se manifiesta con una serie de signos inespecíficos y síntomas generales (Tabla 7). La presencia o ausencia de manifestaciones clínicas está, muchas veces, relacionada con el tiempo de duración de la enfermedad: se observa que, cuando la patología es de larga data, la mayoría de ellas no está presente o lo está en forma muy atenuada.

Las manifestaciones no hematológicas pueden ser causadas por la anemia o, simplemente, por la deficiencia de hierro de por sí, sin necesidad de que se produzca anemia. Tienen la característica de que algunas pueden ser transitorias y reversibles, mientras que otras son permanentes e irreversibles, a pesar del tratamiento, si se producen en etapas precoces de la vida. Si bien, en la actualidad, se acepta que muchas de ellas son, indudablemente, causadas por la deficiencia de hierro, pues hay evidencia científica concluyente que así lo confirma, otras siguen aún siendo motivo de controversia y análisis, ya que no existe todavía evidencia confirmatoria del rol de la deficiencia de hierro en su patogénesis (Tabla 8). ${ }^{40-46}$

TABLA 7. Sintomatología de la anemia

Síntomas generales
• Palidez de piel y mucosas
• Decaimiento
Manifestaciones circulatorias
• Taquicardia
- Hipotensión arterial
Manifestaciones neuromusculares
• Cefalea
• Vensación de mareo y vértigo
• Disminublada
• Cansanción de la capacidad de concentración
Dolor muscular
- Disnea
Manifestaciones respiratorias
Otras manifestaciones
- Nipersensibilidad al frío

TABla 6. Causas de anemia ferropénica

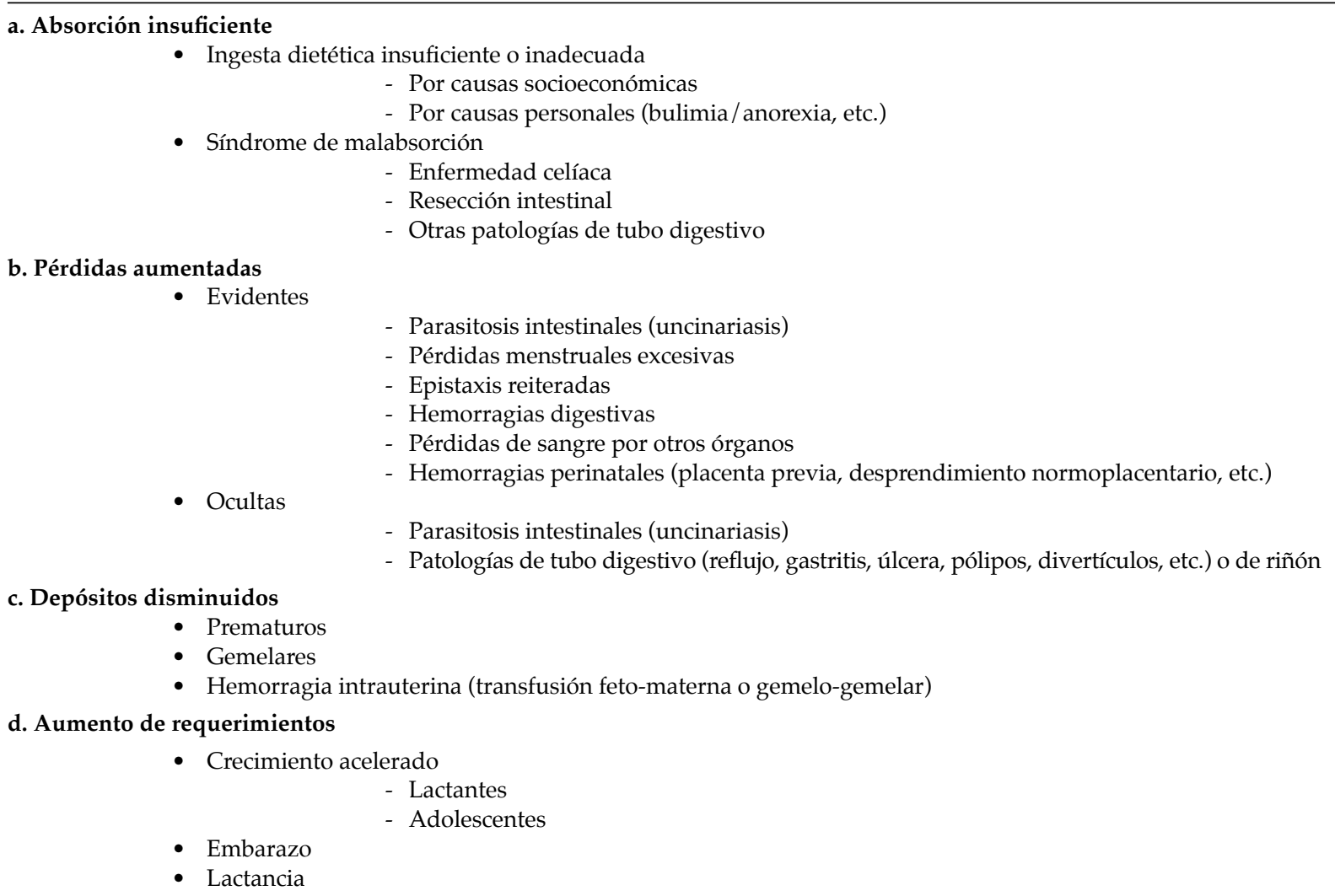




\section{DIAGNÓSTICO}

Debe basarse en lo siguiente: $22,47,48$

1. Interrogatorio. Se debe prestar especial atención a estos puntos:

- Tipo de dieta: duración de la lactancia materna y / o de la ingesta de otras leches o fórmulas, ingesta de carne y alimentos ricos en hierro y otros nutrientes (vitaminas C, A y B12, ácido fólico, zinc), volumen de ingesta diaria de leche, exceso de carbohidratos, etc.

- Antecedentes de prematurez, embarazos múltiples y déficit de hierro en la madre.

- Antecedentes de patología perinatal.

- Pérdidas de sangre: color de heces, epistaxis, disnea, hematuria, hemoptisis, etc.

- Trastornos gastrointestinales: diarrea, esteatorrea, etc.

- Procedencia geográfica: zonas de parasitosis (uncinariasis) endémicas.

- Hábito de pica.

- Suplemento con hierro: cantidad, tiempo, compuesto administrado (sulfato ferroso $\mathrm{u}$ otros).

- Trastornos cognitivos: bajo rendimiento escolar, déficit de atención, etc.

2. Examen físico. La palidez cutáneo-mucosa es el signo principal y se puede también observar retardo del desarrollo pondoestatural, esplenomegalia leve, telangiectasias, alteración de tejidos epiteliales (uñas, lengua, cabello) y alteraciones óseas.

\section{Estudios de laboratorio}

\section{Hemograma}

- Hemoglobina y hematocrito: disminuidos.

- Recuento de reticulocitos: normal. Si está aumentado, se deben investigar pérdidas por hemorragia o posibilidad de otro diagnóstico.
- Recuento de plaquetas: normal o elevado.

- Recuento leucocitario: normal.

- Índices hematimétricos: - Volumen corpuscular medio (VCM): disminuido. Los valores normales durante la infancia son variables y distintos a los del adulto, por lo que, para definir microcitosis, deben tomarse como referencia los valores mostrados en la Tabla 9.11,20

- Concentración de hemoglobina corpuscular media (CHCM): disminuida.

- Amplitud de distribución eritrocitaria (ADE) o red blood cell distribution width (RDW): elevada.

- Morfología eritrocitaria: hipocromía, microcitosis, ovalocitosis, policromatofilia, punteado basófilo (eventualmente).

TABLA 9. Valores normales de volumen corpuscular medio durante la infancia y la adolescencia

\begin{tabular}{|c|c|}
\hline Edad & VCM (fL) \\
\hline Nacimiento & $108(98)$ \\
\hline $1 \mathrm{mes}$ & $104(85)$ \\
\hline 2 meses & $96(77)$ \\
\hline De 3 a 6 meses & $91(74)$ \\
\hline De 6 a 23 meses & $77(70)$ \\
\hline De 2 a 4 años & $79(73)$ \\
\hline De 5 a 7 años & $81(75)$ \\
\hline De 8 a 11 años & $83(76)$ \\
\hline \multicolumn{2}{|l|}{ De 12 a 14 años } \\
\hline \multicolumn{2}{|l|}{ Mujer } \\
\hline De 15 a 17 años & $84(77)$ \\
\hline \multicolumn{2}{|l|}{ De 15 a 17 años } \\
\hline Mujer & $87(79)$ \\
\hline Varón & $86(78)$ \\
\hline \multicolumn{2}{|l|}{ Adulto } \\
\hline Mujer & $90(80)$ \\
\hline Varón & $90(80)$ \\
\hline
\end{tabular}

Los valores entre paréntesis expresan el límite inferior normal (media - 2 DE).

DE: desvío estándar. VCM: volumen corpuscular medio.

TABla 8. Manifestaciones no hematológicas de la deficiencia de hierro

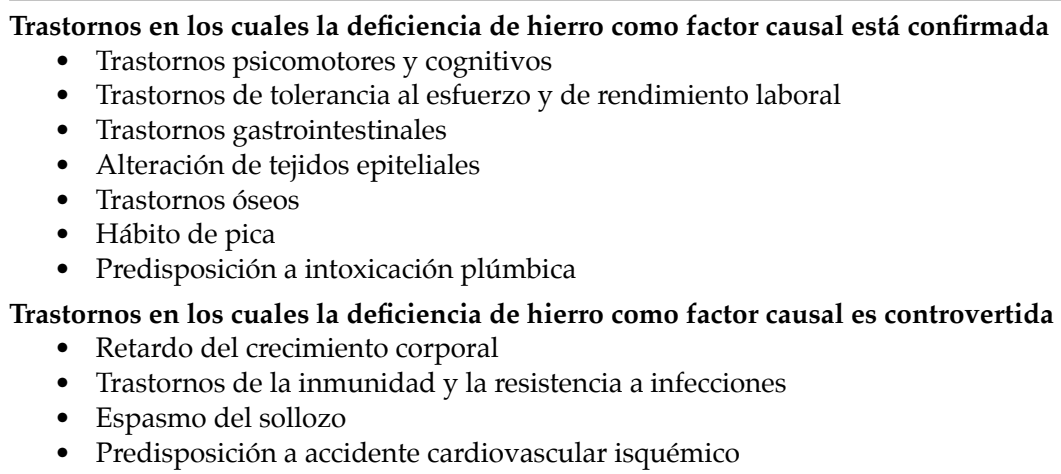




\section{Pruebas que evalúan el estado del hierro}

- Hierro del compartimiento funcional - Ferremia: disminuida.

- Capacidad total de saturación de hierro: aumentada.

- Porcentaje de saturación de la transferrina: disminuido.

- Protoporfirina libre eritrocitaria: aumentada.

- Receptores solubles de transferrina: aumentados.

- Hierro del compartimiento de depósito

- Ferritina sérica: disminuida.

- Hemosiderina en médula ósea: disminuida/ausente.

En la Tabla 10, se muestran los valores de corte recomendados para las determinaciones más habituales. ${ }^{11}$ Con un solo valor alterado, es suficiente para el diagnóstico de ferropenia, pero hay factores que pueden modificar los resultados (Tabla 11), ${ }^{22}$ por lo tanto, no siempre reflejan la deficiencia de hierro y, en ocasiones, el tratamiento debe instaurarse en forma empírica.

4. Prueba terapéutica. Consiste en administrar sulfato ferroso en dosis terapéuticas (3-6 mg/ $\mathrm{kg} /$ día) y evaluar la respuesta eritropoyética. La positividad de la prueba puede evaluarse detectando un pico reticulocitario (recuento $\geq$ $2 \%$ ) a los 5-10 días u observando un aumento de hemoglobina $\geq 1 \mathrm{~g} / \mathrm{dL}$ a los 30 días.
Observación: Las enunciadas son las pruebas disponibles en un laboratorio medianamente equipado. Sin embargo, por lo general, no es necesario recurrir a las que evalúan el estado del hierro, ya que, con el extendido de sangre periférica y los indices hematimétricos, se llega a una fuerte presunción diagnóstica de ferropenia, por lo que se puede intentar una prueba terapéutica. Si se considera conveniente confirmar el diagnóstico mediante pruebas de laboratorio, las más recomendables son porcentaje de saturación, ferritina sérica y protoporfirina libre eritrocitaria. No se recomienda realizar medulograma con la única finalidad de evaluar las reservas de hierro.

\section{DIAGNÓSTICO DIFERENCIAL}

Para el diagnóstico diferencial de anemia ferropénica, véase Anemias microcíticas hipocrómicas: guía de diagnóstico diferencial. En nuestro país, las causas más frecuentes de este tipo de anemias son ferropenia, talasemia menor y anemia de la inflamación. Se debe tener en cuenta que algunas de estas patologías pueden coexistir (p. ej., ferropenia y talasemia menor). ${ }^{22,47}$

\section{DETECCIÓN}

Debido a la alta prevalencia de anemia ferropénica en niños de 6 a 24 meses de edad en nuestro país, se debe pesquisarla mediante la realización sistemática de hemograma y ferritina en el lactante. El estudio se debe realizar entre los

TABla 10. Pruebas confirmatorias para deficiencia de hierro. Valores de corte recomendados

\begin{tabular}{|c|c|c|c|}
\hline Edad & Ferremia* $(\mu \mathrm{g} / \mathrm{L})$ & Saturación de transferrina* $(\%)$ & Ferritina sérica $(\mathrm{ng} / \mathrm{mL})$ \\
\hline De 6 meses a 2 años & --- & --- & $<10$ \\
\hline De 2 a 4 años & $<60$ & $<12$ & $<10$ \\
\hline De 5 a 10 años & $<60$ & $<14$ & $<10$ \\
\hline De 11 a 14 años & $<60$ & $<16$ & $<10$ \\
\hline > 15 años & $<60$ & $<16$ & $<12$ \\
\hline
\end{tabular}

* No se recomiendan estas determinaciones antes de los 2 años de vida por el amplio rango de distribución de los valores normales a esa edad.

TABLA 11. Condiciones que pueden influenciar el resultado de las pruebas confirmatorias para deficiencia de hierro

\begin{tabular}{lll}
\hline Prueba & Elevación & Disminución \\
\hline Ferremia & Toma de muestra en horas de la tarde & Infección/inflamación \\
& Ingesta reciente de alimentos con hierro & \\
Saturación de transferrina & Ingesta reciente de suplemento de hierro & Infección/inflamación \\
Ferritina & Anticonceptivos & Hipotiroidismo \\
& Infección/inflamación & Hipovitaminosis C \\
Protoporfirina libre & Hepatopatía & \\
eritrocitaria & Intoxicación plúmbica & \\
& Anemia hemolítica & \\
& Infección/inflamación & \\
\hline
\end{tabular}


9 y los 12 meses de edad en los recién nacidos de término y entre los 6 y los 9 meses de edad en los prematuros. También se recomienda realizar la pesquisa (hemograma, ferremia, capacidad total de fijación del hierro -total iron binding capacity; TIBC, por sus siglas en inglés-, porcentaje de saturación y ferritina) en adolescentes mujeres luego de que han presentado la menarca.

\section{TRATAMIENTO}

El tratamiento debe apuntar a corregir la anemia, almacenar hierro en depósitos y corregir la causa primaria. En algunos casos, puede ser necesaria una transfusión de glóbulos rojos sedimentados.

\section{a. Corrección de la causa primaria}

Administración de la dieta adecuada, tratamiento de las parasitosis, control del reflujo gastroesofágico, manejo del síndrome de malabsorción, control de pérdidas ocultas, etc.

\section{b. Tratamiento con hierro}

Puede administrarse indistintamente por vía oral o parenteral, ya que la eficacia y el ritmo de ascenso de la hemoglobina son similares. ${ }^{11,49,50}$

- Vía oral: Es de elección. ${ }^{22,47}$ La dosis (calculada en mg de hierro elemental) es $3-6 \mathrm{mg} / \mathrm{kg} /$ día, fraccionada en 1-3 tomas diarias. ${ }^{22,47,48}$ El preparado de elección es el sulfato ferroso, $11,22,47,51-55$ que debe administrarse alejado de las comidas -media hora antes o dos horas después-, ya que muchos alimentos disminuyen la absorción de hierro hasta un $40-50 \% .{ }^{25}$ Cuando la intolerancia al sulfato impide realizar el tratamiento, debe intentarse con otros preparados; de ellos, el que mejor tolerancia presenta es el hierro polimaltosa. ${ }^{56-58} \mathrm{El}$ tiempo de administración es variable: una vez alcanzados valores normales de hemoglobina y hematocrito, debe continuarse, en igual dosis, durante un tiempo igual al que fue necesario para alcanzar la normalización. ${ }^{22,47}$ Esta prolongación del tratamiento sirve para reponer depósitos de hierro. Las complicaciones habituales son intolerancia digestiva (náuseas, constipación, diarrea, vómitos, dolor abdominal) y coloración negruzca de dientes (reversible con la suspensión del tratamiento). ${ }^{59}$
- Vía parenteral: Se utiliza en casos de intolerancia digestiva grave al hierro oral, patología digestiva que contraindique la vía oral o presunción firme de tratamiento oral insuficiente o inadecuado. ${ }^{47,48} \mathrm{La}$ indicación de hierro parenteral deberá ser dada por el médico hematólogo. La dosis total por administrar, para corregir la anemia y reponer los depósitos, se calcula de acuerdo con la siguiente fórmula:22,47,48,60

(Hemoglobina teórica $[\mathrm{g} / \mathrm{dL}]$ hemoglobina real $[\mathrm{g} / \mathrm{dL}]) /$ $100 \times$ volemia $(\mathrm{ml}) \times 3,4 \times 1,5=\mathrm{mg}$ de hierro 3,4: factor de conversión de $\mathrm{g}$ de hemoglobina a mg de hierro. 1,5: hierro de depósitos.

En caso de utilizar la vía intramuscular, la cantidad total de mg de hierro resultante debe fraccionarse en dosis que no excedan de $1,5 \mathrm{mg} / \mathrm{kg} /$ día y administrarse cada 2-3 días. ${ }^{22}$ El preparado recomendado para la administración intramuscular es el hierro dextrano.

Para la administración endovenosa, el paciente debe estar internado. Se recomienda utilizar hierro sacarato. $\mathrm{La}$ cantidad total de $\mathrm{mg}$ de hierro por recibir debe fraccionarse en dosis que no excedan de $2-3 \mathrm{mg} / \mathrm{kg}$ y administrarse cada 2 días. Se debe diluir en solución fisiológica (100 mg en un máximo de $100 \mathrm{~mL}$ de solución fisiológica) y administrar por infusión endovenosa lenta en 1530 minutos (1 mL/minuto). Se debe detener la infusión ante parestesias o dolor torácico. Se deben administrar previamente dosis de prueba en pacientes que lo reciben por primera vez ( $1 \mathrm{~mL}$ y esperar 15 minutos por la ocurrencia de reacciones adversas). ${ }^{61}$ Las complicaciones que pueden observarse con el hierro parenteral son dolor en el sitio de inyección, linfadenitis regional, hipotensión arterial, shock anafiláctico, cefalea, malestar general, urticaria, fiebre, mialgias, artralgias.

- Control del tratamiento y alta hematológica: ${ }^{40}$ Las pautas son similares, independientemente de la vía por la que se administró el tratamiento.

- Los pacientes con hemoglobina $<8 \mathrm{~g} / \mathrm{dL}$ al momento del diagnóstico se controlan 
cada 7 días hasta alcanzar dicho valor y luego cada 30 días hasta alcanzar valores normales para la edad.

- Los pacientes con hemoglobina $\geq 8 \mathrm{~g} / \mathrm{dL}$ al momento del diagnóstico se controlan cada 30 días hasta alcanzar valores normales para la edad.

- Si se utilizó la vía oral, se suspende la administración del hierro luego de haber completado un período de tratamiento igual al que se empleó para normalizar la hemoglobina.

- Se debe analizar dejar al paciente con dosis profilácticas si se considera necesario de acuerdo con su edad, tipo de dieta o patología de base.

- Se recomienda realizar un hemograma de control a los 3 meses de haberse suspendido el tratamiento para detectar posibles recaídas.

- En pacientes que siguen dietas vegetarianas, es conveniente realizar un monitoreo periódico estricto que incluya hemograma y estudio del hierro, debido a la falta de ingesta de alimentos ricos en hierro de alta biodisponibilidad. También es conveniente, en estos casos, estimular el consumo de alimentos fortificados con hierro.

\section{- Causas de falla terapéutica}

La falta de respuesta al tratamiento se puede evidenciar en distintas etapas del tratamiento con hierro (Figura 4). Las causas más frecuentes son las siguientes: ${ }^{22}$ - Incumplimiento del tratamiento: Se debe verificar que el niño haya recibido el preparado recetado, en la dosis correcta, fraccionada según lo indicado, alejado de los alimentos y durante el tiempo indicado. - Prescripción inadecuada: Se debe verificar que los principales aspectos de la prescripción hayan sido correctamente indicados -utilizar de elección sulfato ferroso, en dosis de $3-6 \mathrm{mg} / \mathrm{kg} /$ día, fraccionada en 1-2 tomas diarias, administrado alejado de las comidas ( $1 / 2$ hora antes o 2 h después) y durante el tiempo necesario-.

- Falta de resolución de la causa: En la mayoría de los casos, la causa de la deficiencia de hierro es evidente ya desde el interrogatorio del paciente (parasitosis, hipermenorrea, epistaxis a repetición, prematurez, gemelaridad, etc.) y el fracaso del tratamiento se debe simplemente al hecho de no haber actuado de modo correcto sobre ella. En los otros casos, o sea, pacientes con falla terapéutica que

FIGURA 4. Distintas etapas en las que se puede manifestar falta de respuesta o respuesta inadecuada al tratamiento con hierro

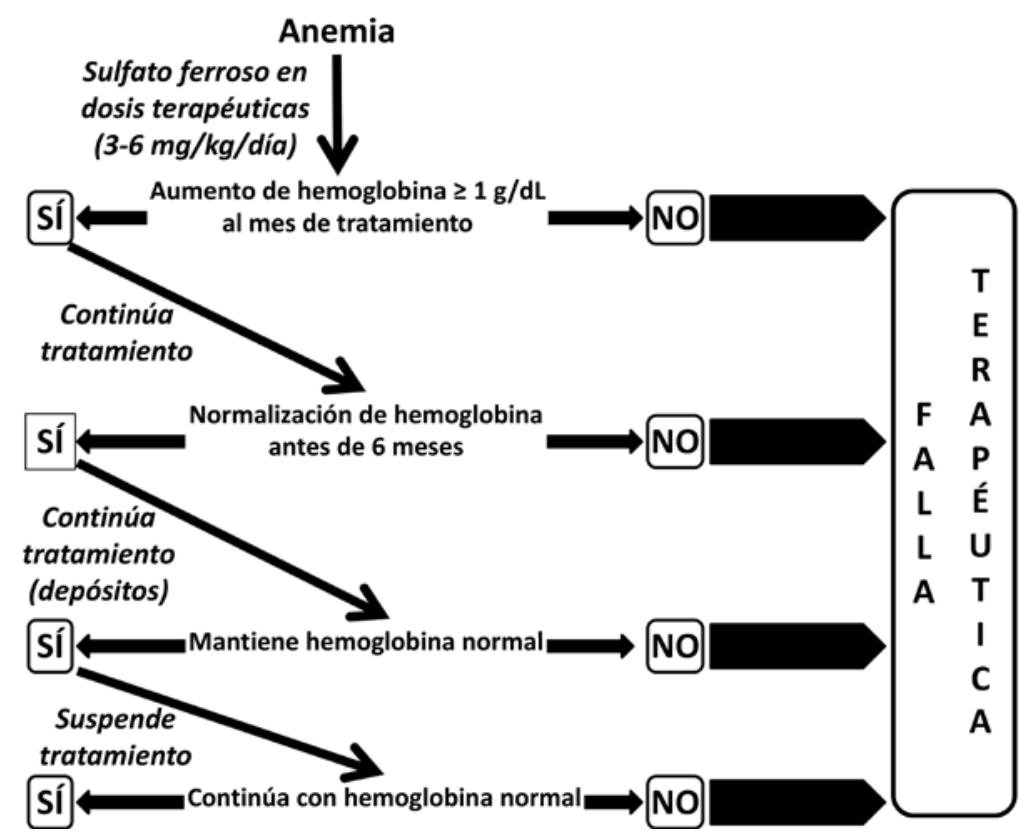


tenían deficiencia de hierro confirmada al momento del diagnóstico inicial, pero sin causa evidente para ella, se debe tratar de detectar la existencia de alguna etiología oculta solicitando, como mínimo, lo siguiente:

- Sangre oculta en materia fecal

- Examen parasitológico de materia fecal

- Orina completa

- Anticuerpos para celiaquía

La asociación entre anemia ferropénica y enfermedad celíaca es muy estrecha. ${ }^{62}$ En el mayor estudio pediátrico publicado hasta la fecha, sobre 434 niños con enfermedad celíaca, se observó que 365 de ellos (84\%) presentaban deficiencia de hierro; más aun, en 169 (39\%), la anemia ferropénica había sido la forma de presentación de la enfermedad. ${ }^{63}$

- Error diagnóstico: Si bien la ferropenia es la principal causa de anemia microcítica en la infancia, frente al fracaso terapéutico, se deben plantear alternativas diagnósticas posibles (véase Anemias microcíticas hipocrómicas: guía de diagnóstico diferencial). - Coexistencia con otra patología hematológica: La anemia ferropénica puede no responder, o hacerlo solo parcialmente, al tratamiento con hierro debido a su coexistencia con otras patologías hematológicas. Las más frecuentes en pediatría son las asociaciones con talasemia menor o con deficiencia de folatos.

- Enfermedad coexistente: La anemia de la inflamación suele acompañar a enfermedades crónicas (colagenopatías, tumores, infecciones, etc.) y puede ser el síntoma único y/o inicial de estas patologías. Es una anemia hipocrómica microcítica indistinguible de la ferropénica por estudios habituales. La determinación de ferremia, capacidad total de saturación y porcentaje de saturación puede servir para diferenciar entre ambas (Figura 5).

\section{c. Transfusión de sangre}

La indicación de transfusión en pacientes con anemia ferropénica es una decisión clínica que debe adoptarse dentro del siguiente contexto: $:^{22,47}$

- Con hemoglobina $\geq 7 \mathrm{~g} / \mathrm{dL}$, no se debe transfundir, excepto para corregir hipoxemia en pacientes con insuficiencia respiratoria.

- Con hemoglobina $<7 \mathrm{~g} / \mathrm{dL}$, se debe transfundir:

- Para corregir descompensación hemodinámica.

- Si coexiste con insuficiencia respiratoria.

- Si hay factores agravantes (desnutrición, infección, diarrea crónica).

- Si la hemoglobina es inferior a $5 \mathrm{~g} / \mathrm{dL}$.

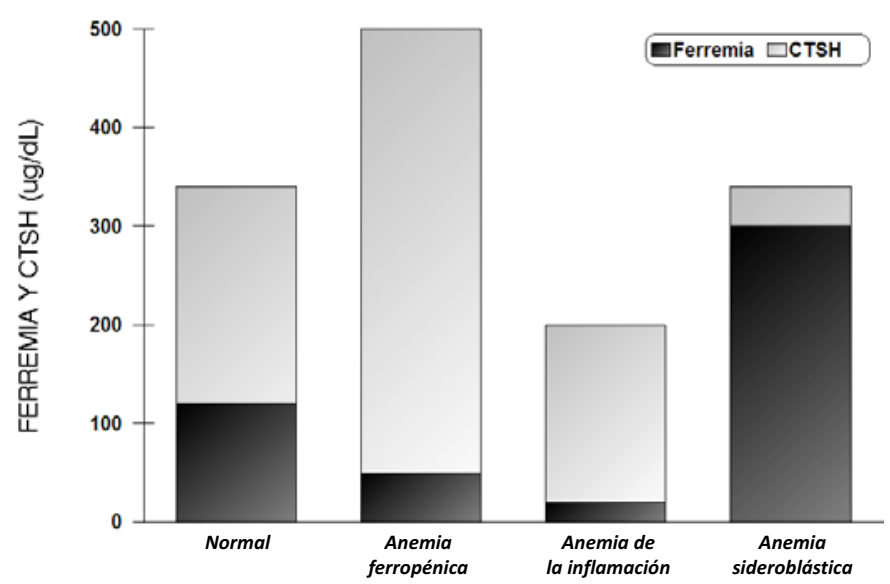

En condiciones normales, la ferremia representa, aproximadamente, el 33\% de la capacidad total de saturación de hierro (CTSH). Tanto en anemia ferropénica (AF) como en anemia de la inflamación (AI), la ferremia está disminuida, pero la CTSH suele estar aumentada en AF y disminuida en AI. Como consecuencia, el porcentaje de saturación va a estar siempre disminuido en AF, mientras que, en $\mathrm{AI}$, puede estar normal o disminuido. 


\section{PROFILAXIS}

Las conductas preventivas que pueden aplicarse están en relación con las condiciones biológicas, ambientales y socioeconómicas de la madre y el niño. Comprenden varios aspectos: ${ }^{22}$

\section{a. Conductas perinatales}

- Sostén de la adecuación del hierro corporal en la embarazada.

- Incremento del hierro de depósito al nacer. Se recomienda la ligadura tardía del cordón umbilical (1-3 minutos luego del nacimiento), con lo cual se logra aumentar los depósitos de hierro corporal en, aproximadamente, $30 \%$ y disminuir la incidencia de anemia ferropénica. ${ }^{64-67}$ Se debe tener en cuenta que los riesgos de hiperbilirrubinemia y de síndrome de policitemia/hiperviscosidad en el neonato, así como los de hemorragia posparto grave en la madre, no han sido aún suficientemente evaluados. ${ }^{66,67}$

\section{b. Conductas alimentarias}

- Se debe promover la lactancia materna exclusiva para los primeros 6 meses de vida.

- Se debe favorecer la alimentación complementaria oportuna y adecuada con la introducción, a los 6 meses, de papillas junto con alimentos de consistencia similar ricos en hierro de alta biodisponibilidad. Se debe enfatizar la importancia de la lactancia materna y, una vez comenzada la alimentación con sólidos, la introducción precoz de alimentos ricos en hierro $(30 \mathrm{~g} /$ día, equivalentes a, aproximadamente, 2 cucharas soperas), ${ }^{68-70}$ siguiendo los lineamientos sobre biodisponibilidad que se muestran en la Figura $2 .{ }^{22}$
- Fortificación de alimentos. Numerosos alimentos y algunas leches de vaca están suplementados con hierro de aceptable biodisponibilidad. ${ }^{28,31,33,68,69,71-73}$ Se debe tratar de evitar el uso de leche de vaca en menores de 12 meses. Si bien la fortificación de alimentos es considerada una herramienta eficaz para la prevención de la ferropenia, los estudios a gran escala no han podido establecer claramente su utilidad..$^{74,75}$ Sin embargo, en algunas investigaciones con programas focalizados y controlados, se ha mostrado efectiva para disminuir la anemia en la población infantil. ${ }^{6}$ Se debe tener en cuenta que la ingesta indiscriminada de alimentos fortificados en niños no carenciados de hierro conlleva el riesgo potencial de desencadenar enfermedad por sobrecarga de hierro (hemocromatosis). ${ }^{74}$

\section{c. Conductas farmacológicas}

- Se debe administrar suplemento con hierro medicinal a los siguientes grupos de riesgo: $22,48,76-78$

- Prematuros

- Gemelares

- Niños con bajo peso de nacimiento

- Niños que hayan sufrido hemorragias en el período perinatal

- Niños de término alimentados con leche de vaca sin fortificación

- Niños de término alimentados a pecho que reciben alimentación complementaria inadecuada con bajo contenido de hierro - Niños con patologías que impliquen malabsorción o pérdida crónica de hierro - Adolescentes con pérdidas menstruales excesivas

- Adolescentes embarazadas

TABLA 12. Dosis profilácticas de hierro para administrar en los distintos grupos de riesgo

\begin{tabular}{lccc}
\hline Grupo & Dosis & Inicio & Duración \\
\hline RN de término & $1 \mathrm{mg} / \mathrm{kg} /$ día & 2 meses & Hasta los 12 meses \\
RN pretérmino $(1500-2000 \mathrm{~g})$ & $2 \mathrm{mg} / \mathrm{kg} /$ día & 1 mes & Hasta los 18 meses \\
RN pretérmino $(750-1500 \mathrm{~g})$ & $3-4 \mathrm{mg} / \mathrm{kg} /$ día & Durante el primer mes & Hasta los 18 meses \\
RN pretérmino $(<750 \mathrm{~g})$ & $5-6 \mathrm{mg} / \mathrm{kg} /$ día & Durante el primer mes & Hasta los 18 meses \\
RN con bajo peso de nacimiento & $1-2 \mathrm{mg} / \mathrm{kg} /$ día & Durante el primer mes & Hasta los $12-18$ meses \\
Mujeres adolescentes & $60-80 \mathrm{mg} /$ día & \multicolumn{2}{c}{ Durante 3 meses consecutivos por año } \\
Adolescentes embarazadas & $60 \mathrm{mg} /$ día & Al comienzo del 2do trimestre & Hasta el final del embarazo \\
\hline
\end{tabular}

RN: recién nacido. 
- Las dosis por administrar se muestran en la Tabla 12.22,47,48,76,79

- El preparado de elección que se aconseja utilizar es el sulfato ferroso. $22,47,48,53,76,77$

- No deben recibir suplementos de hierro los niños de término, eutróficos, alimentados con fórmulas en forma regular $(100 \mathrm{~g}$ de polvo/día, equivalentes a $600 \mathrm{~mL} /$ día). ${ }^{80}$

- Desparasitación: La OMS recomienda la desparasitación masiva en zonas de alta prevalencia de uncinariasis, ${ }^{81}$ como el Noreste Argentino. Según norma del Ministerio de Salud de la Nación (2005), se deben administrar 2 dosis de mebendazol por año a niños de 2-14 años. ${ }^{82}$

\section{REFERENCIAS}

1. De Benoist B, McLean E, Egli I, Coswell M. Worldwide Prevalence of Anaemia 1993-2005. WHOGlobalDatabase on Anaemia. World Health Organization, Centers for Disease Control and Prevention, 2008.

2. WHO/UNICEF/ONU. Iron deficiency anemia assessment, prevention, and control. Geneva. World Health Organization; 2001.

3. ENNyS. Encuesta Nacional de Nutrición y Salud. Documento de resultados. Ministerio deSalud, Presidencia de la Nación. Buenos Aires, 2006.

4. Calvo EB, Gnazzo N. Prevalence of iron deficiency in children aged 9-24 mo from a large urban area of Argentina. Am J Clin Nutr 1990;52:534-40.

5. Morasso MC, Molero J, Vinocur P, Acosta L, Paccussi N, Raselli $S$, et al. Deficiencias de hierro y de vitamina A y prevalencia de anemia en niños y niñas de 6 a 24 meses de edad en Chaco, Argentina. Arch Latinoam Nutr 2003;53:219.

6. Varea A, Malpeli A, Etchegoyen G, Vojkovic M, Disalvo L, Apezteguía M, et al. Short-term evaluation of the impact of a food program on the micronutrient nutritional status of Argentinean children under the age of six. Biol Trace Elem Res 2011;143(3):1337-48.

7. Hurrell RF, Reddy MB, Juillerat M, Cook JD. Meat protein fractions enhance nonheme iron absorption in humans. $J$ Nutr 2006;136:2808-12.

8. Boletín. ProAPS-REMEDIAR. Año 2 n. ${ }^{\circ}$ 9, abril 2004. ISSN1668-2831.

9. Christensen L, Squassero Y, Cuesta CB. Anemia y adherencia a la suplementación oral con hierro en una muestra de niños usuarios de la red de salud pública de Rosario, Santa Fe. Arch Argent Pediatr 2013;111:288-94.

10. Dallman PR, Siimes MA, Stekel A. Iron deficiency in infancy and childhood. Am J Clin Nutr 1980;33:86-118.

11. Dallman PR, Yip R, Oski FA. Iron deficiency and related nutritional anemias. En: Hematology of Infancy and Childhood. Nathan DG, Oski FA (eds.). Philadelphia, W.B. Saunders, 1993.p.413-50.

12. Rao R, Georgieff MK. Iron in fetal and neonatal nutrition. Semin Fetal Neonatal Med 2007;12:54-63.

13. Kumar A, Rai AK, Basu S, Dash D, Singh JS. Cord blood and breast milk iron status in maternal anemia. Pediatrics 2008;121:e673-e677.

14. Andelman MB, Sered BR. Utilization of dietary iron by term infants. Am J Dis Child 1966;111:45-55.

15. Fomon SJ, Ziegler EE, Nelson SE, Edwards BB. Cow milk feeding in infancy: gastrointestinal blood loss and iron nutritional status. J Pediatr 1981;98:540-5.

16. NUTRIABA. Encuesta Nutricional a Niñas / os Menores de 6 años de la Provincia de Buenos Aires. Programa Materno Infantil. Ministerio de Salud de la Provincia de Buenos Aires, 1999.

17. Donato H, Buys MC. Eritropoyesis normal. En: Donato H, Rapetti C, eds. Anemias en Pediatría. Buenos Aires; Fundasap; 2005.p.11-32.

18. Lundstrom U, Siimes MA, Dallman PR. At what age does iron supplementation become necessary in low-birthweight infants? J Pediatr 1977;91:878-83.

19. Saarinen UM, Siimes MA. Developmental changes in red blood cell counts and indices of infants after exclusion of iron deficiency by laboratory criteria and continuous iron supplementation. J Pediatr 1978;92:412-6.

20. Dallman PR, Sijmes MA. Percentile curves for hemoglobin and red cell volume in infancy and childhhod. J Pediatr.1979;94:26-31.

21. Dallman PR. Progress in the prevention of iron deficiency in infants. Acta Paediatr Scand (suppl.) 1990;365:s28-s37.

22. Donato H, Rapetti C, Crisp R, Buys MC. Anemias carenciales. En: Donato H, Rapetti C (eds). Anemias en Pediatría. Buenos Aires: Fundasap; 2005.p.39-86.

23. Norby A. Iron absorption studies in iron deficiency. Scand J Haematol 1974;20(suppl.):1-15.

24. Beard JL, Piñero DJ. Metabolismo del hierro. En: O'Donnell AM, Viteri FE, Carmuega E (eds): Deficiencia de Hierro. Desnutrición Oculta en América Latina. Buenos Aires, CESNI, 1997.p.13-48.

25. Hallberg L, Bjorn-Rasmussen E, Ekenved G, Garby L, Rossander L, Pleehachinda R, et al. Absorption from iron tablets given with different types of meals. Scand J Haematol 1978;21:215-22.

26. Lynch SR. Absorción de hierro: Interacción con otros nutrientes. En:O'Donnell AM, Viteri FE, Carmuega E (eds.): Deficiencia de Hierro. Desnutrición Oculta en América Latina. Buenos Aires, CESNI; 1997.p.49-66.

27. Saarinen UM, Siimes MA, Dallman PR. Iron absorption in infants: High bioavailability of breast milkiron as indicated by the extrinsic tag method of iron absorption and by the concentration of serum ferritin. J Pediatr 1977;91:36-9.

28. McMillan JA, Oski FA, Lourie G, Tomarelli RM, Landaw SA. Iron absorption from human milk, simulated human milk, and proprietary formulas. Pediatrics 1977;60:896-900.

29. Hicks PD, Zavaleta N, Chen Z, Abrams SA, Lonnerdal B. Iron deficiency, but not anemia, upregulates iron absorption in beast-fed Peruvian infants. J Nutr 2006;136:2435-8.

30. Domellöff $M$. Iron requirements, absorption and metabolism in infancy and childhood. Curr Opin Clin Nutr Metab Care 2007;10:329-35.

31. Ríos E, Hunter RE, Cook JD, Finch CA. The absorption of iron as supplements in infant cereal and infant formulas. Pediatrics 1975;55:686-9.

32. Saarinen UM, Siimes MA. Iron absorption from infant formula and the optimal level of iron supplementation. Acta Paediatr Scand 1977;66:719-25.

33. Saarinen UM, Siimes MA. Iron absorption from breast milk, cow's milk, and iron-supplemented formula: an opportunistic use of changes in total body iron determined by hemoglobin, ferritin and body weight in 132 infants. Pediatr Res 1979;13:143-6.

34. Garry PJ, Owen GM, Hooper EM, Gilbert BA. Iron absorption from human milk and formula with and without iron supplementation. Pediatr Res 1981;15:822-8.

35. Hopkins D, Emmett P, Steer C, Rogers I, Noble S, Emond A. Infant feeding in the second 6 months of life related to iron status: an observational study. Arch Dis Child 2007;92: 850-4. 
36. Kemna EHJM, Tjalsma H, Willems HL, Swinkels DW. Hepcidin: from discovery to differential diagnosis. Haematologica 2008;93:90-7.

37. American Academy of Pediatrics, Committee on Nutrition: Iron supplementation for infants. Pediatrics 1976;58:765-9.

38. National Research Council. Recommended dietary allowances. 9th. ed., Washington, DC: National Academy Press, 1980.

39. Dietary Reference Intakes: Elements. NAS. IOM. Food and Nutrition Board. 2011.

40. Donato H, Rapetti MC, Crisp RL, Buys MC. Anemia ferropénica y trastornos hereditarios del metabolismo del hierro. En: Donato H, Rapetti MC, Crisp RL (eds). Anemias en Pediatría. Buenos Aires: Ed. Journal; 2014.p.35-80.

41. Lozoff B. Iron deficiency and child development. Food Nutr Bull 28(suppl):S560-S571.

42. Bhatia MS, Singhal SPK, Dhar NK, Nigam VR, Malik SC, Mullick DN. Breath holding spells: an analysis of 50 cases. Indian Pediatr 1990;27:1073-9.

43. Daoud AS, Batieha A, Al-Sheyyab M, Abuekteish F, Hijazi S. Effectiveness of iron therapy on breath-holding spells. J Pediatr 1997;130:547-50.

44. Mocan H, Yildiran A, Orhan F, Erduran E. Breath holding spells in 91 children and response to treatment with iron. Arch Dis Child 1999;81:261-2.

45. Ziaullah NawazS, ShahS, Talaat A. Iron deficiency anemia as a cause of breath holding spells. J Postgrad Med Inst 2005;19:171-4.

46. Maguire JL, deVeber G, Parkin PC. Association between iron-deficiency anemia and stroke in young children. Pediatrics 2007;120:1053-7.

47. Comité Nacional de Hematología. Anemia ferropénica. Guía de diagnóstico y tratamiento. Arch Argent Pediatr 2009:107:353-61.

48. Donato H. Anemias: detección, tratamiento y profilaxis. En: Comité Nacional de Pediatría General Ambulatoria. Boggiano E, Breitman F, Andrade M (eds.): Manual para la Supervisión de la Salud de Niños, Niñas y Adolescentes. Buenos Aires, Fundasap, 2010.p.265-72.

49. McCurdy PR. Oral and parenteral iron therapy. JAMA 1965;191:859-70.

50. Finch CA. Drugs effective in iron deficiency and other hypochromic anemias. En: Goodman LS, Gilman A, eds.: The Pharmacologic Basis of Therapeutics, New York, The Macmillan Company, 1980.p.1315-29.

51. Arvas A, Gur E. Are ferric compounds useful in treatment of iron deficiency anemia? Turk J Pediatr 2000;42:352-3.

52. Kavakli K, Yilmaz D, Cetinkaya B, et al. Safety profiles of $\mathrm{Fe}^{2+}$ and $\mathrm{Fe}^{3+}$ oral preparations in the treatment of iron deficiency anemia in children. Pediatr Hematol Oncol 2004;21:403-10.

53. Lanzkowsky P.En Lanzkowsky P(ed.). Manual of Pediatric Hematology and Oncology. Burlington, USA: Elsevier Inc.; 2005.p.31-46.

54. Ozsoylu S. Treatment of iron deficiency anemia. Pediatr Hematol Oncol 2005;22:645-6.

55. Donato H, Rapetti MC, Moran L, Cavo M. Comparación entrehierro polimaltosa y sulfato ferroso para el tratamiento dela anemia ferropénica: estudio prospectivo aleatorizado. Arch Argent Pediatr 2007;105:491-7.

56. Jacobs P, Wood L, Bird AR. Better tolerance of iron polymaltose complex compared with ferrous sulphate in the treatment of anaemia. Hematology 2000;5:77-83.

57. Walter T,Zacarías I, Yañez CG. Tolerance and acceptability in infants of iron polymaltose complex. Ars Medicine 2005;9:428-31.

58. Toblli JE, Brignoli R. Iron (III)-hydroxide polymaltose complex in iron deficiency anemia. Review and meta- analysis. Arzneimittel-Forschung (Drug Research) 2007;57(6a):431-8.

59. Reeves JD, Yip R. The lack of adverse side effects of oral ferrous sulphate therapy in the 1-year old infants. Pediatrics 1985;75:352-55

60. Smith CH. La anemia ferropénica. En: Smith $\mathrm{CH}$ (ed.): Hematología Pediátrica, Salvat Ed, 1973.p.188-219.

61. www.garrahan.gov.ar/vademecum.

62. Winter S, Halsey C. Should children presenting with iron deficiency anaemia be screened for coeliac disease? Arch Dis Child 2014;99:180-2.

63. Kochhar R, Jain K, Thapa BR, Rawal P, Khaliq A, Kocchar $\mathrm{R}$, et al. Clinical presentation of celiac disease among pediatric compared to adolescent and adult patients. Indian J Gastroenterol 2012;31:116-20.

64. Chaparro CM, Neufeld LM, Tena Alavez G, Eguia-Liz Cedillo R, Dewey KG. Effect of timing of umbilical cord clamping on iron status in Mexican infants: a randomised controlled trial. Lancet 2006;367:1997-2004.

65. Ceriani Cernadas JM, Carroli G, Pellegrini L, Otaño L, Ferreira M, Ricci C, et al. The effect of timing of cord clamping on neonatal venous hematocrit values and clinical outcome at term: a randomized, controlled trial. Pediatrics 2006;117:e779-e886.

66. McDonald SJ, Middleton P. Effect of timing of umbilical cord clamping of term infants on maternal and neonatal outcomes. Cochrane Database Syst Rev 2008;CD004074.

67. Ceriani Cernadas JM, Carroli G, Pellegrini L, Ferreira M, Ricci A, Casas O, et al. Efecto del clampeo demorado del cordón umbilical en la ferritina sérica a los seis meses de vida. Estudio clínico aleatorizado. Arch Argent Pediatr 2010;108:201-8.

68. Pizarro F, Yip R, Dallman PR, Olivares M, Hertrampf E, Walter T. Iron status with different infant feeding regimens: Relevance to screening and prevention of iron deficiency. J Pediatr 1991;118:687-92.

69. Comité de Nutrición. Guía de Alimentación para Niños Sanos de 0 a 2 Años. Sociedad Argentina de Pediatría, 2001.

70. Engelmann MD, Sandstrom B, Michaelsen KF. Mean intake and iron status in late infancy: an intervention study. Pediatr Gastroenterol Nutr 1998;26:26-33.

71. Rapetti MC, Donato H, De Galvagni A, Lubovitsky M, Lanzilotta M, Trepacka E, et al. Correction of iron deficiency with an iron-fortified fluid whole cow's milk in children: Results of a pilot study. J Pediatr Hematol Oncol 1997;19:1926.

72. Donato H, Rapetti MC, de Galvagni A, Lubovitsky M, Lanzilotta M, Trepacka E, et al. Treatment of iron deficiency with an iron fortified, fluid, cow's milk associated to low doses of iron. Comparison with the usual ferrous sulfate therapy. Br J Haematol 1998;102:40.

73. Olivares M. Bioavailability of microencapsulated ferrous sulfate in milk. Nutrition 2002;18:285-6.

74. Dewey KG. Increasing iron intake of children through complementary foods. Food Nutr Bull 2007;28(suppl):s595-s609.

75. Lynch S, Stolzfus R, Rawat R. Critical review of strategies to prevent and control iron deficiency in children. Food Nutr Bull 2007;28(suppl):s610-s619.

76. Guideline: Daily Iron Supplementation in Adult Women and Adolescent Girls. Geneva: World Health Organization; 2016.

77. Guideline: Daily Iron Supplementation in Infants and Children. Geneva: World Health Organization; 2016.

78. Calvo E. Anemia por deficiencia de hierro en niños y embarazadas. Boletín PROAPS - REMEDIAR, Año 1, n. ${ }^{\circ}$ 4, octubre 2003.

79. Ministerio de Salud de la Nación. Nutrición y Embarazo. 
s82 / Arch Argent Pediatr 2017;115 Supl 4:s68-s82 / Subcomisiones, Comités y Grupos de Trabajo

Recomendaciones en nutrición para los equipos de salud. Dirección Nacional de Maternidad e Infancia. Buenos Aires: Ministerio de Salud, 2012

80. Domellof M, Braegger C, Campoy C, Colomb V, Decsi T, et al. ESPGHANCommitttee on Nutrition. Iron requirements of infants and toddlers. J Pediatr Gastroenterol Nutr 2014;58: 119-29.
81. World Health Organization. Report of the WHO Informal Consultation on the Use of Chemotherapy for the Control of the Morbidity Due to Soil-Transmitted Nematodes in Humans. Ginebra, Suiza:OMS1996. (WHO/CTD/SIP/96.2

82. Guía para el Equipo de Salud. Programa Nacional de Desparasitación Masiva (1. ${ }^{\text {era }}$ edición). Programa Remediar. Ministerio deSalud de la Nación. Buenos Aires. Febrero 2005. 